\title{
Kissing nevus of the penis: a case report and dermatoscopic findings*
}

\author{
Nivea Godinho ${ }^{1}$ \\ Arnaldo Luiz Flávio Schaefer ${ }^{3}$
}

\author{
Gisele Alborghetti Nai ${ }^{2}$ \\ Luiza Vasconcelos Schaefer ${ }^{4,5}$
}

DOI: http:/ / dx.doi.org/10.1590/abd1806-4841.20175574

\begin{abstract}
Divided nevus, also known as kissing nevus, is a rare variant of congenital melanocytic nevi in which there are two adjacent nevi in areas of the body that undergo embryonic cleavage. The original description of this type of lesion was on the eyelid. The location on the penis is even rarer, with only 17 case reports in the literature so far, and only one of them described the dermoscopic findings. We report the case of a patient with divided nevus of the penis and its clinical, dermoscopic and histopathological features.
\end{abstract}

Keywords: Dermoscopy; Melanocytes; Nevus; Nevus, pigmented; Penis

\section{INTRODUCTION}

Divided nevus, also known as kissing nevus, is a clinical variant of congenital melanocytic nevi in which there are two adjacent nevi that involve areas of the body that undergo embryonic cleavage. ${ }^{1-4}$ The original description of this type of lesion was on the eyelids in 1908, and since then few cases have been reported. The same mechanism for melanocytic lesions was already described in other rarer locations, such as fingers and penis..$^{1-6}$ So far, there have been 17 cases reporting lesions on the penis. ${ }^{5}$ We present a case of divided nevus on the penis and its dermoscopic and histopathological findings.

\section{CASE REPORT}

A 9-year-old boy was referred to the dermatology department with two dark, asymptomatic lesions on the penis. The macules were seen by his mother after balano-preputial exposure when he was six years old. The patient was previously healthy, non-circumcised, with no history of local trauma nor changes on the le- sions over this period of time. Physical examination revealed brown macules on the right lateral aspect of the glans and prepuce of the penis, almost symmetrical in both sides of the coronal sulcus. At rest, the size of the lesion on the prepuce was smaller than the lesion on the glans due to the primary contraction of the skin on the prepuce. Upon retraction of the foreskin, the lesions appeared as a mirrored image (Figure 1). Dermoscopy of both macules revealed melanocytic lesions in a compound pattern. In the periphery, there was fine pigment network and darkened globules of multiple sizes in the centre. The lesions were separated by a thin streak of non-pigmented skin on the prepuce and it was possible to observe a few globules on the coronal sulcus (Figure 2). Histopathology of both lesions revealed a proliferation of densely pigmented melanocytes with abundant cytoplasm, along the dermal-epidermal junction and forming nests in the papillary dermis suggestive of a compound melanocytic nevus (Figure 3).

Study submitted on 12.01 .2016

Approved by the Advisory Board and accepted for publication on 21.04.2017

* Study conducted at Hospital Regional de Presidente Prudente "Dr. Domingos Leonardo Cerávolo" - Universidade do Oeste Paulista (HRPP- Unoeste) Presidente Prudente - SP, Brazil.

Financial Support: None

Conflict of Interests: None

Post-graduation Program in Dermatology - Hospital Regional de Presidente Prudente "Dr. Domingos Leonardo Cerávolo" - Universidade do Oeste Paulista (HRPP- Unoeste) - Presidente Prudente (SP), Brazil.

Department of Pathology, Faculdade de Medicina of the Universidade do Oeste Paulista (Unoeste) - Presidente Prudente (SP), Brazil.

3 Department of Urology - Hospital Regional de Presidente Prudente “Dr. Domingos Leonardo Cerávolo" - Universidade do Oeste Paulista (HRPP- Unoeste) Presidente Prudente (SP), Brazil.

4 Department of Dermatology, Faculdade de Medicina da Universidade do Oeste Paulista (Unoeste) - Presidente Prudente - SP, Brazil.

5 Department of Dermatology - Hospital Regional de Presidente Prudente "Dr. Domingos Leonardo Cerávolo" - Universidade do Oeste Paulista (HRPPUnoeste) - Presidente Prudente (SP), Brazil.

(C)2017 by Anais Brasileiros de Dermatologia 


\section{DISCUSSION}

The divided nevus was first described in 1908 by Van Michael Paul, who reported two melanocytic lesions in opposite margins of the upper and lower eyelid that, upon closing the eyes, looked like a single lesion. ${ }^{3,5}$ In 1919, it was called divided nevus or kissing nevus by Fuchs. The first case on the penis was described by Desruelles in 1998, and since then few cases were reported in the world. ${ }^{1,3,5}$ It is suggested that the lesion is initially single and that it divides during the development of the external genitalia. ${ }^{5,7}$ Between the $11^{\text {th }}$ and $14^{\text {th }}$ week of gestation, two invaginations appear on the penis. ${ }^{3}$ The glandular epithelium forms the glandular urethra and the preputial epithelium divides and forms the glans and the prepuce. Derruelles advocates that the nevus is caused by the migration of melanoblasts before completing the invagination of the preputial epithelium and that after completing the embryonic division each nevus continues to develop independently. Kono suggests that the migration of melanoblasts occurs soon after the embryonic separation of the glans and prepuce.,3,4

Clinically, they are well-defined oval-shaped pigmented macules ranging from brown to black, with a smooth surface. They present as two mirrored lesions, symmetrical in relation to the coronal sulcus. ${ }^{1,3}$ The site of predilection is almost always the dorsal
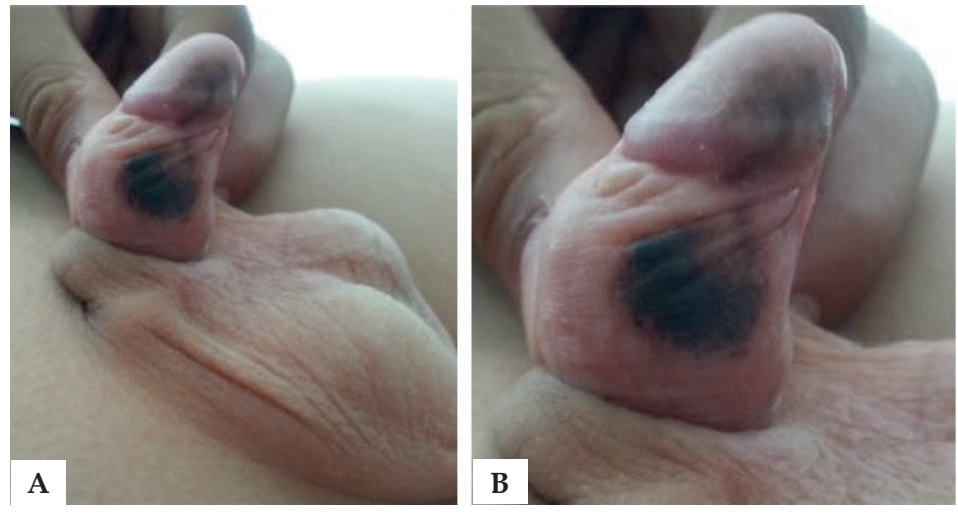

Figure 1:

A, B, C: Brown macules on the lateral aspect of the glans and prepuce of the penis, almost symmetrical on both sides of the coronal sulcus

Figure 2:

Dermoscopy of the macules revealed melanocytic lesions in a compound pattern. In the periphery, there was fine pigment network and in the center, dark globules of different sizes. A: lesion on the glans; B: lesion on the prepuce
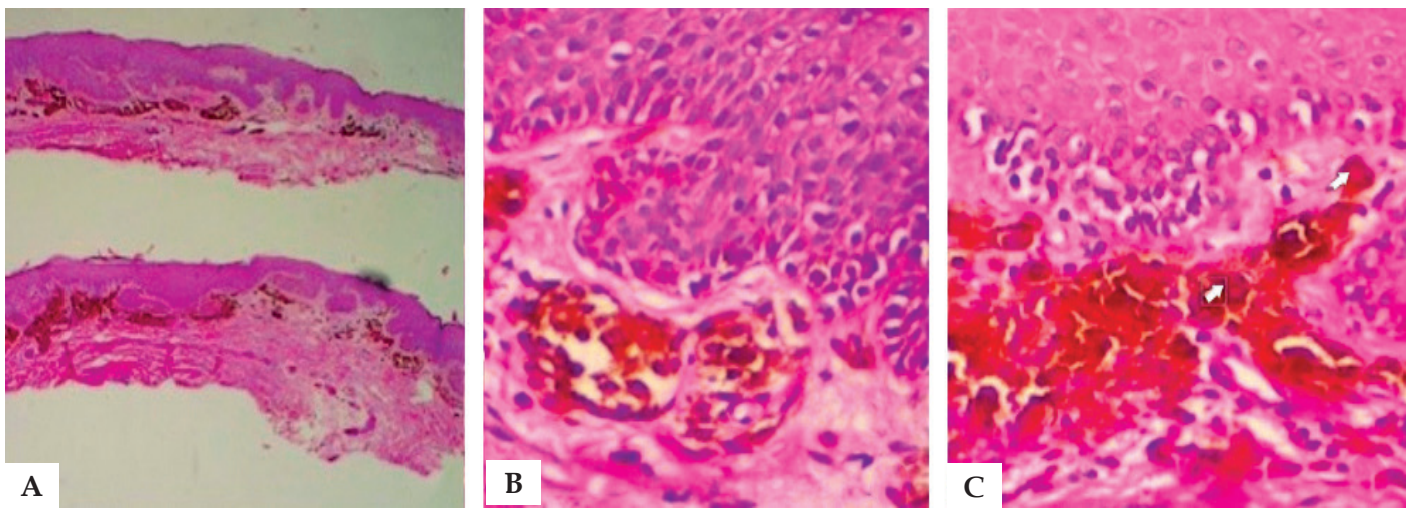

Figure 3: Photomicroscopy of the glans skin. A: Proliferation of densely pigmented melanocytes along the dermal-epidermal junction and papillary dermis (Hematoxylin \& eosin, X100); B: Proliferation of densely pigmented melanocytes along the dermal-epidermal junction and forming nests in the papillary dermis (Hematoxylin \& eosin, X400); C: Melanocytes with evident nuclei (arrows) (Hematoxylin \& eosin, X400) 
and dorso-lateral penis, what can be explained by the fact that the epithelial invagination of the dorsal aspect precedes the ventral. ${ }^{3}$ The diagnosis of divided nevus is clinical, and the dermoscopy and histopathology are important ancillary tests for the diagnosis and patient follow-up.

Dermoscopy has been very useful in the analysis of cutaneous and mucosal pigmented lesions, helps long-term follow-up, and avoids unnecessary biopsy and surgery. Only one publication has reported dermoscopic findings of two patients with divided nevus on the penis thus far. Large globules and compound patterns were demonstrated, formed by a peripheral pigment network and an area with central globules. ${ }^{7}$ Similarly to the literature, this patient also had the compound pattern in both macules as seen on dermoscopy.

On the histopathology, most lesions present as intradermal or compound melanocytic nevi. ${ }^{3}$ Only one case of divided nevus on the penis with malignant transformation has been reported. The patient was diagnosed at the age of 30 years, had no family history of melanoma, and presented with a rapidly enlarging lesion and changes of pigmentation. However, the dermoscopy of the lesion was not described. ${ }^{8}$

Malignant melanoma of the penis is very rare and corresponds to less than $2 \%$ of all primary malignant lesions of the penis. In the majority of cases, it is localized on the glans (55\%), followed by the prepuce $(28 \%)$, penis shaft $(9 \%)$ and urethral meatus $(8 \%)$. The majority of melanoma cases of the penis occurs in the $6^{\text {th }}$ and $7^{\text {th }}$ decades of life and these lesions have a poor prognosis. Due to the potential of malignant transformation of divided nevus, we highlight the importance of follow-up of the patients with clinical and dermoscopic examination in order to speed treatment in case there is any change of the lesion.

The differential diagnosis should be made with melanotic macule, fixed drug eruption and melanoma (specially in adults). ${ }^{7}$

Considering the benign nature of divided nevus, treatment is optional. ${ }^{7}$ The therapy should focus on functional and aesthetic results. ${ }^{2,3,5}$ Some authors performed surgical treatment with satisfactory results. The nevi are exercised and the repair is done using a graft from labial mucosa or remaining foreskin after postectomy. ${ }^{1-3,5,7}$ However, for large nevi, there is the concern of aesthetic or functional damage. For this reason, the use of Nd:YAG laser may a therapeutic alternative. ${ }^{1-3,5}$

The proper knowledge about this nevus in combination with the dermoscopic features enables patient follow-up without surgical treatment, specially in case of children, to avoid aesthetic and functional impairment resulting from a scar or a surgical defect.

In our case, the patient is undergoing dermatologic and urologic follow-up as an outpatient, with programmed dermoscopy and photographic documentation of the lesion every three months.]

\section{REFERENCES}

1. Hardin CA, Tieu KD. Divided or kissing nevus of the penis. Dermatol Online J. 2013;19:20027.

2. Wang S, Zhou M, Qiao J. Kissing nevus of the penis. Report of two cases and review of the literature. An Bras Dermatol. 2014;89329-31.

3. Yun SJ, Wi HS, Lee JB, Kim SJ, Won YH, Lee SC. Kissing nevus of the penis. Ann Dermatol. 2011;23:512-4.

4. Kono T, Nozaki M, Kikuchi Y, Erçöçen AR, Hayashi N, Chan HH, et al. Divided naevus of the penis: a hypothesis on the embryological mechanism of its development. Acta Derm Venereol. 2003;83:155-6.

5. Li ZB, Liu T, Zhang QG, Hu JT. Treatment of Divided Nevus of the Penis with Circumcision and Free Inner Prepuce Grafting. Plast Reconstr Surg Glob Open. 2015;3:e389.

6. Nizawa M, Masahashi T, Maie 0 , Takahashi S. A case of solitary mastocytoma suggesting a divided form of mast cell nevus. J Dermatol. 1989;16:402-4.

7. Mendes CP, Samorano LP, Alessi SS, Nico MM. Divided naevus of the penis: two paediatric cases with dermoscopic findings. Clin Exp Dermatol. 2014;39:728-30.

8. Egberts F, Egberts JH, Schwarz T, Hauschild A.. Kissing Melanoma or Kissing Nevus of the Penis? Urology. 2007;69:384.e5-7.

\section{MAILING ADDRESS: \\ Nivea Godinho Alves de Souza \\ Rua José Bongiovani, 1297 \\ 19050-680 Presidente Prudente, SP \\ Brazil \\ E-mail:nivea60@hotmail.com}

How to cite this article: Alves NG, Nai GA, Schaefer ALF, Schaefer LV. Kissing nevus of the penis: a case report and dermatoscopic findings. An Bras Dermatol. 2017;92(5 Suppl 1): 95-7. 